\title{
NOTAS
}

\section{INVENTARIO DE LAS ARAÑAS (ARACHNIDA: ARANEAE) \\ DE LA RESERVA ECOLÓGICA LIMONES-TUABAQUEY, SIERRA DE CUBITAS, CAMAGÜEY, CUBA}

\author{
Giraldo Alayón García ${ }^{1}$, Alexander Sánchez-Ruiz ${ }^{2}$ Yulianis Martín Castejón ${ }^{3}$, Maylen Ramírez Ruiz \\ y Franklin Cala Riquelme 5
}

\begin{abstract}
1. Museo Nacional de Historia Natural, Ciudad de La Habana. Cuba.
2. Centro Oriental de Ecosistemas y Biodiversidad. Santiago de Cuba. Cuba.

3. Centro de Investigaciones de Medio Ambiente Camagüey, Camagüey. Cuba.

4. Empresa para la Protección de la Flora y la Fauna, Nuevitas, Camagüey. Cuba.

5. Empresa para la Protección de la Flora y la Fauna, Santiago de Cuba. Cuba.
\end{abstract}

\section{RESUMEN}

Se realiza un inventario rápido de las arañas de la Reserva Ecológica Limones-Tuabaquey, Sierra de Cubitas, Camagüey, Cuba. En total, se registran para el área protegida 71 especies, agrupadas en 59 géneros y 26 familias. Se registra por primera vez para Cuba y las Antillas Mayores la especie Homalometa nigritarsi Simon, 1897 (Tetragnathidae) y se amplia además la distribución conocida de cinco especies: Ariamnes mexicanus Exline y Levi, 1962, Anisaitis squamata Bryant, 1940 (Salticidae), Scytodes blanda Bryant, 1940 (Scytodidae), Theridion antillanum Simon, 1894 (Theridiidae) y Episinus gratiosus Bryant, 1940 (Theridiidae). Se determinan 17 especies endémicas, las cuales se consideran objetos de conservación del área protegida, particularmente Anisaitis squamata Bryant, 1940 y Scytodes blanda Bryant, 1940; especies raras que sólo se conocen de pocas localidades en el archipiélago cubano.

\section{ABSTRACT}

A rapid biological inventory of spiders from Limones-Tuabaquey Ecological Reserve was conducted. 71 species, 59 genera and 26 families are reported for this protected area. The species Homalometa nigritarsi Simon, 1897 (Tetragnathidae) is reported for the first time for Cuba and Greater Antilles. The geographic limits of other five species are enlarged: Ariamnes mexicanus Exline y Levi, 1962, Anisaitis squamata Bryant, 1940 (Salticidae), Scytodes blanda Bryant, 1940 (Scytodidae), Theridion antillanum Simon, 1894 (Theridiidae) y Episinus gratiosus Bryant, 1940 (Theridiidae). Seventeen endemic species were identified and considered conservation targets for this protected area; especially Anisaitis squamata Bryant, 1940 and Scytodes blanda Bryant, 1940, rare species that are only known for few localities in the Cuban archipelago.

Key words: Inventory, spiders, Sierra de Cubitas, Cuba.

La Reserva Ecológica Limones-Tuabaquey se encuentra situada a $32 \mathrm{~km}$ al norte de la ciudad de Camagüey, en la Sierra de Cubitas. Esta área protegida, con una extensión de $2,700 \mathrm{~km}^{2}$, presenta valores naturales y culturales de gran importancia para la conservación de la biodiversidad cubana (Díaz et al., 2006). Entre los principales valores naturales se encuentran el Cerro Tuabaquey (335 msnm), el Cerro Mirador de Limones (309 msnm), el Paso Paredones y el Hoyo de Bonet.

La mayor contribución al estudio de la fauna de invertebrados en esta área protegida fue realizada en septiembre del 2002, cuando un equipo de investigadores cubanos y norteamericanos realizaron un inventario biológico rápido en la Sierra de Cubitas (Díaz et al., 2006). En este estudio sólo se inventariaron cuatro grupos de invertebrados (moluscos, cucarachas, mariposas y hormigas). Posteriormente, en junio de 2007, el Centro Oriental de Ecosistemas y Biodiversidad y el Centro de Investigaciones de Medio Ambiente de Camagüey realizaron un segundo inventario aún no publicado, profundizando en algunos de los grupos ya trabajados, pero incluyendo además los escorpiones y los himenópteros.

La fauna de arañas de esta área protegida hasta el momento no se conocía. Algunas colectas aisladas se habían efectuado, pero nunca antes se habían publicado registros de especies de arañas. Con excepción de Microsa cubitas Alayón y Platnick, 1993, un endémico local descrito de un área cercana a la Reserva Ecológica Limones-Tuabaquey (Alayón y Platnick, 1993), el resto de las especies registradas en este trabajo se dan a conocer por primera vez para la zona.

Durante los días del 6 al 12 de mayo de 2008 realizamos un tercer inventario en el área protegida, esta vez enfocado únicamente en la fauna de arañas. Se visitaron cinco localidades dentro de la Reserva 
Ecológica (Fig. 1): 1. Cerro Tuabaquey $\left(21^{\circ} 34^{\prime} 33^{\prime \prime} \mathrm{N}-77^{\circ} 45^{\prime} 05^{\prime \prime} \mathrm{W}\right) ; 2$. Estación Ecológica Limones-Tuabaquey $\left(21^{\circ} 35^{\prime} 50^{\prime \prime} \mathrm{N}-77^{\circ} 47^{\prime} 29^{\prime \prime} \mathrm{W}\right)$; 3. Senderos de las epífitas $\left(21^{\circ} 35^{\prime} 55^{\prime \prime} \mathrm{N}\right.$ $\left.77^{\circ} 47^{\prime} 06^{\prime \prime} \mathrm{W}\right)$; 4. Paso Paredones $\left(21^{\circ} 36^{\prime} 02^{\prime \prime} \mathrm{N}-77^{\circ} 47^{\prime} 08^{\prime \prime} \mathrm{W}\right)$; 5 . Hoyo de Bonet $\left(21^{\circ} 36^{\prime} 55^{\prime \prime} \mathrm{N}\right.$ $\left.77^{\circ} 47^{\prime} 03^{\prime \prime} \mathrm{W}\right)$. Se revisó exhaustivamente en la vegetación, en el suelo y bajo piedras y troncos, además se realizó la colecta con red entomológica en la vegetación baja y se colocaron siete trampas de caída.

Para la Reserva Ecológica Limones-Tuabaquey son registradas un total de 71 especies de arañas, agrupadas en 59 géneros y 26 familias; lo cual representa el $12 \%$ de las especies conocidas en Cuba. De estas especies, 17 resultaron endemismos del archipiélago cubano. Con este trabajo se registra por primera vez para Cuba y las Antillas Mayores la especie Homalometa nigritarsi Simon, 1897 (Tetragnathidae), conocida anteriormente de las Antillas Menores, México y Panamá (Levi, 1986). Esta especie fue descrita originalmente de San Vicente, Antillas Menores, de ocho hembras, un macho y nueve juveniles (Simon, 1897); también se ha encontrado en Trinidad y Tobago (Sewal y Alayón García, inédito). No es una especie abundante y se sabe poco sobre su historia natural; los ejemplares colectados (hembras) se encontraron en el envés de hojas, debajo de una tela laminar, guardando los huevecillos.

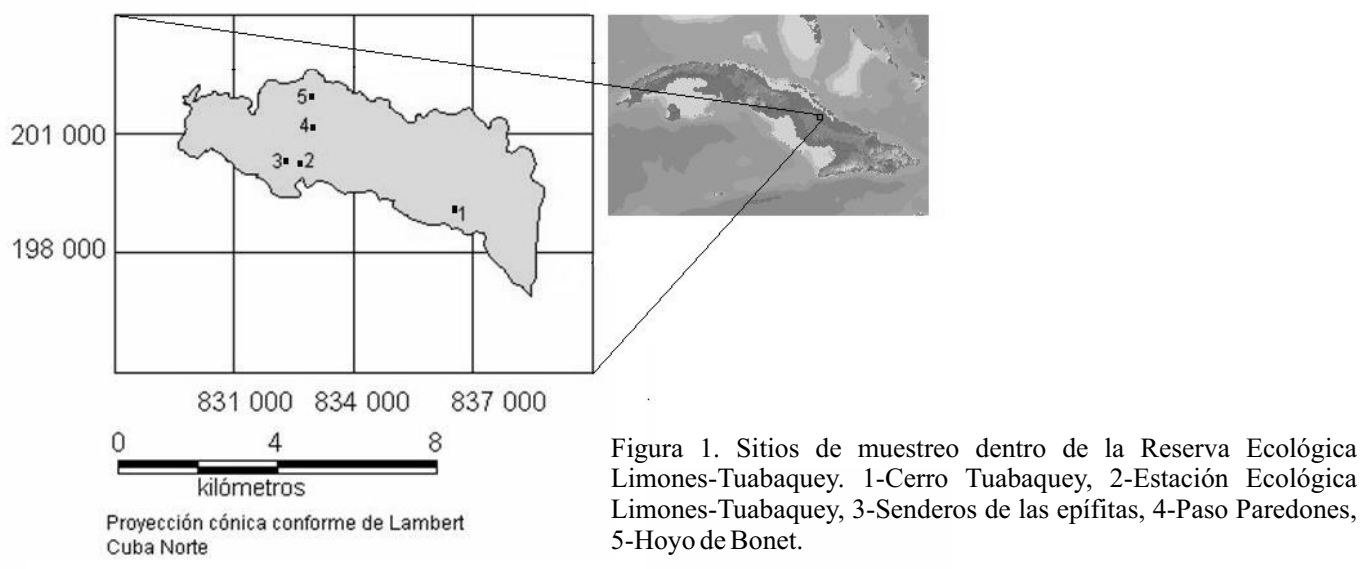

Se amplía, además, la distribución conocida de cinco especies, entre ellas Ariamnes mexicanus Exline y Levi, 1962 (Theridiidae), araña muy rara conocida en Cuba de sólo dos ejemplares depositados en la colección personal del primer autor (GAG) y colectados hace más de 30 años por el entomólogo Fernando de Zayas en la localidad de Soroa, Pinar del Río, Cuba. Afortunadamente en los primeros días de muestreo fueron capturados dos ejemplares hembras de esta especie en el Cerro Tuabaquey y Paso Paredones respectivamente, lo que permitió observar y fotografiar los ejemplares y las ootecas en su ambiente natural (Fig.2). Se amplia la distribución de Anisaitis squamata Bryant, 1940 (Salticidae) endémico conocido anteriormente del occidente del país en las provincias de Cienfuegos y La Habana (Alayón García, 2002). Scytodes blanda Bryant, 1940 (Scytodidae) es un endémico raro, conocido de pocas localidades en las provincias Guantánamo, Santiago de Cuba, Las Tunas, Sancti Spiritus, Cienfuegos, Pinar del Río e Isla de la Juventud (Alayón García, 2002). Así como los Theridiidae Episinus gratiosus Bryant, 1940 y Theridion antillanum Simon, 1894, ambas especies antillanas raras, conocidas de muy pocos ejemplares. Se destacan varios ejemplares pertenecientes a los géneros Chrysso (Tetragnathidae), Theridula (Theridiidae) y Modisimus (Pholcidae) que no se corresponden con ninguna de las especies descritas para estos géneros, por lo que se necesitan mayores estudios.

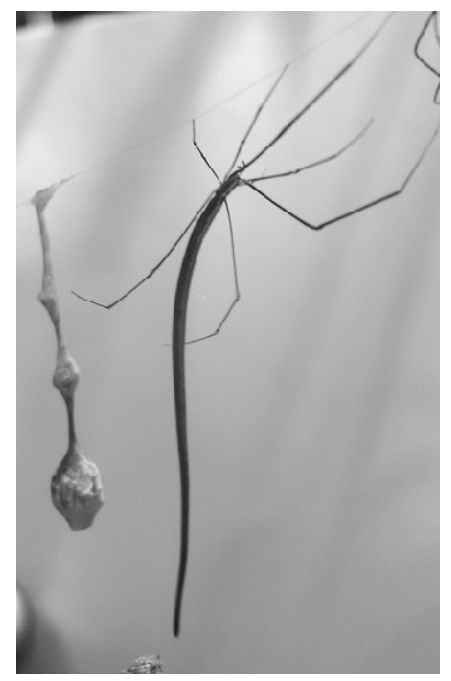

Figura 2. Hembra de Ariamnes mexicanus Exline y Levi, 1962 en su tela con saco de huevos; fotografiada en Cerro Tuabaquey, Sierra de Cubitas, Camagüey, Cuba. 
Como objetos de conservación de la Reserva Ecológica Limones-Tuabaquey se seleccionaron las 17 especies endémicas presentes en el área protegida, particularmente Anisaitis squamata Bryant y Scytodes blanda Bryant, especies raras que sólo se conocen de pocas localidades en el archipiélago cubano.

Finalmente, deseamos agradecer a todo el personal de la Estación Ecológica Limones-Tuabaquey y del Centro de Investigaciones de Medio Ambiente de Camagüey por su apoyo y preocupación durante los días del inventario.

\section{LISTA DE ESPECIES DE LA RESERVA ECOLOGICA LIMONES-TUABAQUEY}

Marcadas con asterisco las especies endémicas de Cuba; los números al final del nombre específico indican los sitios de colecta: 1. Cerro Tuabaquey; 2. Estación Ecológica Limones-Tuabaquey; 3. Senderos de las epífitas; 4. Paso Paredones; 5 . Hoyo de Bonet.

\section{DIPLURIDAE}

\section{INFRAORDEN MYGALOMORPHAE}

1. Ischnothele longicauda Franganillo, 1930: 1

BARYCHELIDAE

2. Trichopelma sp. *: 3

THERAPHOSIDAE

3. Citharacanthus spinicrus (Latreille, 1819): 2, 3

4. Phormictopus sp.: 4

5. Phormictopus nesiotes Chamberling, $1917 *: 2$

\section{INFRAORDEN ARANEOMORPHAE}

\section{FILISTATIDAE}

6. Kukulcania hibernalis (Hentz, 1842) : 1, 2

\section{SICARIIDAE}

7. Loxosceles cubana Gertsch, $1958: 2$

SCYTODIDAE

8. Scytodes blanda Bryant, $1940 *: 4,5$

9. Scytodes fusca Walckenaer, $1837: 2,5$

10. Scytodes longipes Lucas, 1844: 1, 2

11. Scytodes robertoi Alayón, 1977 *: 1

OCHYROCERATIDAE

12. Theotima sp.: 1

PHOLCIDAE

13. Bryantina incerta (Bryant, 1940) *: 3, 5

14. Leptopholcus delicatulus Franganillo, $1930 *$ *: 5

15. Modismus sp. *: 3, 5

15. Physocyclus globosus (Taczanowski, 1873): 1, 2

OONOPIDAE

17. Ischnothyreus peltifer (Simon, 1891): 3, 4

18. Triaeris sp.: 5

MIMETIDAE

19. Mimetus sp.: 4

OECOBIIDAE

20. Oecobius concinus Simon, 1892: 1, 2

DEINOPIDAE

21. Deinopis lamia MacLeay, 1839: 4 


\section{ULOBORIDAE}

22. Miagrammopes latens Bryant, 1936 *: 2, 4

23. Philoponella semiplumosa (Simon, 1893): 2, 3, 5

24. Uloborus trilineatus Keyserling, 1882: 3, 4, 5

THERIDIIDAE

25. Achaearaneae tesselata (Keyserling, 1884): 1, 3

26. Argyrodes elevatus Taczanowski, 1872: 1, 3

27. Ariamnes mexicanus Exline y Levi, 1962: 1, 4

28. Chrysso sp. *: 1

29. Episinus gratiosus Bryant, 1940: 3

31. Latrodectus mactans (Fabricius, 1775): 2

32. Nesticodes rufipes (Lucas, 1846): 1

33. Theridion sp.1: 2

34. Theridion sp.2: 3

35. Theridion antillanum Simon, 1894: 2, 3

36. Theridion evexum Keyserling, 1884: 4, 5

37. Theridula sp.*: 3

38. Tidarren sisyphoides (Walckenaer, 1841): 1, 2, 5

MYSMENIDAE

39. Mysmenopsis tibialis (Bryant, 1940) *: 1

\section{LINYPHIIDAE}

40. Ceratinopsis ruberrima Franganillo, 1926 *: 1, 2, 4

41. Florinda coccinea (Hentz, 1850): 2, 4

TETRAGNATHIDAE

42. Chrysometa linguiformis (Franganillo, 1930): 2, 3

43. Homalometa nigritarsis Simon: 1, 3, 4

44. Leucauge argyra (Walckenaer, 1841): 2, 4, 5

45. Leucauge regny (Simon, 1897): 2

\section{ARANEIDAE}

46. Alcimosphenus licinus Simon, 1895: 4

47. Argiope argentata (Fabricius, 1775): 4

48. Cyclosa caroli (Hentz, 1850): 2

49. Cyclosa walckenaeri (O. P. Cambridge, 1863): 2, 5

50. Eriophora ravilla (C. L. Koch, 1841): 5

51. Gasteracantha cancriformis (Linnaeus, 1767): 2

52. Micrathena cubana (Banks, 1909) *: 5

53. Verrucosa arenata (Walckenaer, 1841): 1

54. Witica crassicaudus (Keyserling, 1865): 5

LYCOSIDAE

55. Hogna sp.: 1

OXYOPIDAE

56. Peucetia viridans (Hentz, 1832): 4

CTENIDAE

57. Ctenus sp. *: 3

58. Ctenus vernalis Bryant, 1940 *: 4, 5

59. Cupiennius cubae Strand, 1910: 3 


\author{
ANYPHAENIDAE \\ 60. Hibana tenuis (L. Koch, 1866): 5 \\ SELENOPIDAE \\ 61.Selenops aissus Walckenaer, 1837: 2, 5 \\ SPARASSIDAE \\ 62. Heteropoda venatoria (Linnaeus, 1767): 2 \\ 63. Pseudosparianthis cubana Banks, 1909: 2 \\ THOMISIDAE \\ 64. Misumenops bellulus (Banks, 1896): 2, 4 \\ SALTICIDAE \\ 65. Anasaitis squamata (Bryant, 1940) *: 4, 5 \\ 66. Hentzia antillana Bryant, 1940: 3, 4 \\ 67. Hentzia sp.:2 \\ 68. Lyssomanes antillanus Peckham y Wheeler, 1889: 2 \\ 69. Menemerus bivittatus (Dufour, 1831): 2 \\ 70. Neon nigriceps Bryant, 1940*: 1, 5 \\ 71. Phidippus regius (C. L. Koch, 1846): 1
}

\title{
LITERATURA CITADA
}

Alayón García, G. 2002. Las arañas endémicas de Cuba (Arachnida: Araneae). Revista Ibérica de Aracnología 2: 1-48.

Alayón García, G. y Platnick, N. I. 1993. Review of the Cuban Ground Spiders of the family Gnaphosidae (Araneae: Gnaphosoidea). American Mus. Novitates, 3062: 1-9.

Díaz, L., M., W. S. Alverson, A. Barreto y T. Wachter. 2006. Cuba: Camagüey, Sierra de Cubitas. Rapid Biological Inventories Report 08. The Field Museum, Chicago.

Levi, H.W. 1986. The Neotropical orb-weaver genera Chrysometa and Homalometa (Araneae: Tetragnathidae). Bull. Mus. comp. Zool. Harv. 151:91-215.

Sewal, E. y Alayón García, G. (inédito). Arañas (Arachnida: Araneae) de Trinidad y Tobago, Antillas Menores.

Simon, E. 1897. On the spiders of the island of St Vincent. III.. Proc. zool. Soc. Lond. 1897: 860-890. 